\title{
DESARROLLO PERSONAL Y PROFESIONAL DE MAESTRAS DE EDUCACIÓN PRIMARIA: APORTES DE LA ORIENTACIÓN \\ PRIMARY SCHOOL TEACHERS' PERSONAL AND PROFESSIONAL DEVELOPMENT: \\ CONTRIBUTIONS FROM COUNSELING
}

\author{
SECCIÓN ESPECIAL \\ Volumen 15, Número 1 \\ Enero - Abril \\ pp. 1-29
}

Este número se publicó el $1^{\circ}$ de enero de 2015

DOI: dx.doi.org/10.15517/aie.v15i1.17624

Ericka Isabel Jiménez Espinoza

Revista indizada en REDALYC, $\underline{\text { SCIELO }}$

Revista distribuida en las bases de datos:

CATÁLOGO DE LATINDEX, IRESIE, CLASE, DIALNET, DOAJ, E-REVIST@S, SHERPA/ROMEO, QUALIS, MIAR

Revista registrada en los directorios:

ULRICH'S, REDIE, RINACE, OEI, MAESTROTECA, PREAL, CLACSO 


\title{
DESARROLLO PERSONAL Y PROFESIONAL DE MAESTRAS DE EDUCACIÓN PRIMARIA: APORTES DE LA ORIENTACIÓN \\ PRIMARY SCHOOL TEACHERS' PERSONAL AND PROFESSIONAL DEVELOPMENT: CONTRIBUTIONS FROM COUNSELING
}

\begin{abstract}
Ericka Isabel Jiménez Espinoza ${ }^{1}$
Resumen: Este documento rescata algunos de los resultados más importantes de una experiencia investigativa de tipo cualitativa, con un método fenomenológico. Se llevó a cabo con un grupo de maestras de Educación Primaria de una institución educativa subvencionada, perteneciente a la Dirección Regional de Educación de San José Norte, Costa Rica. La técnica, acorde con los propósitos y naturaleza del estudio que facilitó la recolección de la información, fue la entrevista en profundidad y se plantearon preguntas claves que dirigieron el proceso de estudio. La validación de la información se realizó mediante la técnica de discusión grupal. Un hallazgo importante, giró en torno al traslado que se hace de elementos del desarrollo personal hacia el desarrollo profesional de mujeres docentes en una cultura patriarcal. Finalmente, se presentan aportes relevantes de la disciplina de Orientación sobre esta temática.
\end{abstract}

Palabras clave: DESARROLLO PERSONAL, DESARROLLO PROFESIONAL, DOCENCIA, GÉNERO, ORIENTACIÓN

\begin{abstract}
This paper refers to the main findings of a qualitative research experience in which a phenomenological method was used. The study was undertaken with primary school teachers from one of the state-subsidised institutions of the North San José Educational Region. Several key questions guided the research process, such as: What personal and professional self-concept do the primary school teachers in the group have? What do the teachers consider is needed to enhance their own personal and professional development? What can Counseling provide to promote personal and professional development of primary school teachers? The technique, according to the purpose and nature of the study the best suited for information gathering was the in-depth interview. Group discussion was the technique used for validation of the data. An important finding is that in a patriarchal culture, women teachers transfer elements of their personal development to the area of their professional development. Finally, it was presented the Counseling discipline's relevance related to these situations.
\end{abstract}

Key words: PERSONAL DEVELOPMENT, PROFESSIONAL DEVELOPMENT, TEACHING, GENDER AND COUNSELING

${ }^{1}$ Docente de la Escuela de Orientación y Educación Especial de la Universidad de Costa Rica.

Dirección electrónica: ericka.jimenez@ucr.ac.cr

Documento recibido: 2 de junio, 2014

Enviado a corrección: 21 de agosto, 2014

Aprobado: 8 de diciembre, 2014 


\section{Introducción}

Las personas poseen múltiples habilidades que les permiten desenvolverse en los diferentes ambientes, sean estos sociales, laborales u otros. La sociedad genera cambios constantes y demandas, que les exigen a las personas hacerles frente. Algunas desarrollan sus habilidades personales con seguridad y creatividad, otras lo hacen con mayor dificultad, por lo que han de requerir apoyo e intervención de profesionales de distintas disciplinas, como lo hace la Orientación ante esta y otras situaciones relacionadas con el desarrollo personal y social.

Con el propósito de atender las necesidades en los contextos educativo y empresarial, se han diseñado diversos programas y entrenamientos para docentes, personal de salud mental, gerentes y en general, hacia personas cuyo trabajo requiere de un variado y buen manejo de habilidades personales y sociales. Para ejemplificar, se han impulsado intervenciones de carácter preventivo en instituciones que albergan poblaciones susceptibles de presentar dificultades interpersonales (Hidalgo y Abarca, 2000).

Resulta importante enfocar esfuerzos hacia el fortalecimiento de los recursos personales y así afrontar las demandas sociales. Tonon (2003) indica que para toda persona profesional de ayuda, las cargas laborales suelen ser muy altas, producto de las mismas características de sus funciones que son dirigidas por lo general, hacia el servicio y el apoyo, aspectos que en ocasiones les impiden alcanzar las expectativas depositadas en su trabajo. Esta autora cita a Maslach (1976), quien diera a conocer el término "bournout" como un síndrome que afecta la calidad de vida, el cual perturba especialmente a profesionales de ayuda, tanto en el área de salud como de la educación.

Por su parte, Aron y Milicic (2004) argumentan que el desarrollo personal ha demostrado ser una de las variables de mayor significado para el mejoramiento de la calidad de la educación. Igualmente, ha mostrado su utilidad en la prevención de problemas de salud mental de docentes y de estudiantes. También se le postula como un factor predictivo de logros y satisfacciones en la vida de las personas.

Lo anterior se relaciona con el favorecimiento del desarrollo personal y laboral de mujeres profesionales que trabajan en el sistema educativo costarricense, lo que repercute directamente en el mejoramiento de la calidad de vida; máxime, porque no existen estudios dirigidos a poblaciones adultas con el tema en cuestión. Al ser la persona docente un paradigma para las niñas y los niños, es necesario propiciar estilos de vida saludables en el contexto educativo. Al educador le corresponde la formación no sólo académica, sino 
también la socio-emocional, porque las y los estudiantes se encuentran en una etapa esencial de su desarrollo, lo que implica, de parte de la persona profesional, un compromiso complejo, demandante y de constante introspección de sus propias capacidades y condiciones personales y profesionales.

Para la disciplina de la Orientación, es fundamental trabajar con las personas en el proyecto y sentido de sus vidas, tomando en cuenta su entorno social y laboral desde la condición femenina. Por ello, es necesario trabajar de forma preventiva para favorecer el desarrollo personal y las relaciones interpersonales en grupos de docentes, de manera que puedan hacer frente a sus propios ajustes personales y profesionales, producto de los cambios constantes en el entorno, como también para prevenir posibles situaciones que provoquen desajustes en su desarrollo personal y social.

Este artículo, recoge algunos aportes que son resultado de una experiencia investigativa de tipo cualitativa, efectuada con un grupo de maestras de educación primaria durante el año 2007, cuyo propósito general, es promover el desarrollo personal y profesional de maestras de educación primaria, mediante el aporte de la disciplina de la Orientación. Además, en esta oportunidad y valiéndose de algunos de los aportes de esta investigación, se pretende ofrecer posibles respuestas a interrogantes como las siguientes:

- ¿Qué concepto personal y profesional de sí mismas, poseen un grupo de maestras de educación primaria?

- ¿Qué aspectos son requeridos según las maestras, para favorecer el propio desarrollo personal y profesional?

- ¿Qué aportes puede brindar la Orientación para el desarrollo personal y profesional de maestras de educación primaria?

\section{Acercamiento teórico}

\subsection{La mujer en la adultez}

Algunos estudios argumentan que a la edad adulta no se le atribuye la importancia que merece. Autores como Smelzer y Erikson (1983) consideran que esta etapa ha sido considerada, erróneamente, como un paso caracterizado por la quietud con cambios ocasionales, cuando en realidad es lo contrario, es una época en la que el cambio es continuo, con pequeñas pausas de quietud y con una sobrecarga de tareas que requieren de mucha energía de la persona, es un paso de gran productividad que necesita trabajo en distintos aspectos de la vida. 
Según Bonilla (1992) existen ciertas tareas que son definidas de acuerdo con el tipo de cultura de la sociedad a la que se pertenezca, a la edad y al género, una vez que son cumplidas, la persona entra en otro período o fase de la vida. La importancia de las labores en la etapa adulta, se encauzan a señalar una guía hacia la cual cada persona se dirige en un momento de su vida, de acuerdo con la forma en que utiliza e integra las destrezas que previamente ha adquirido.

Por su parte, Bustamante (1997) argumenta, a partir de su enfoque en las relaciones genéricas en la cultura patriarcal, que la condición de la mujer, históricamente, puede ser descrita desde la palabra crisis, debido a que se caracteriza por el cambio, el conflicto y la tensión. Según esta autora, la crisis ha sido compañera inseparable de la mujer en la búsqueda de su propia identidad. La evolución de su condición se ha caracterizado por una constante lucha contra estereotipos y prejuicios sociales que han tratado de sojuzgarla sin tregua.

Al tomar en cuenta los aspectos psicosociales, existen diferencias biológicas entre hombres y mujeres, que repercuten, sin duda, en el comportamiento. La socialización, influye en buena medida en el modo de actuar, pensar y sentir de cada quien. Rodríguez (2009) argumenta que como producto de esas diferencias, en ocasiones, a la mujer se le percibe como un ser subordinado, el hecho de que su cuerpo sea más débil físicamente que el del hombre y que, por su naturaleza esté destinado a la procreación, ha sido siempre el argumento para justificar su dominación.

Es necesario que tanto hombres como mujeres, sean conscientes de la forma en que socialmente han sido definidos, consecuencia de las diferencias y con ello, de la necesidad de favorecer cambios en sus propios procesos de vida a partir del reconocimiento de posibles desigualdades, más importante aún del redescubrimiento de sus fortalezas personales. Es preciso que las mujeres adultas partan de reconocerse a sí mismas, mediante la comprensión de sus propias vidas, de su realidad genérica y etapa vital, lo que les permitirá desarrollar o fortalecer habilidades que favorezcan el desarrollo personal para enfrentar los múltiples retos experimentados por las mujeres encargadas de la docencia y por ende, les puedan proporcionar una mejor calidad de vida.

\subsection{Conceptualización del desarrollo personal femenino}

El área personal constituye un espacio de aprendizaje constante y continuo que se experimenta durante la vida de cada persona. Si bien es muy individual, se alimenta de las 
distintas áreas en que las personas se desenvuelven. Existen diversos conceptos de desarrollo personal; a continuación, se destacan aquellas visiones relacionadas con los propósitos que se pretenden aclarar, además se muestran sus distintos componentes y las implicaciones para la construcción de la identidad de la mujer adulta en la sociedad patriarcal.

En un estudio realizado, se conceptualiza el desarrollo personal de la siguiente manera:

El desarrollo personal es un concepto que se refiere a la actualización de todas las potencialidades afectivas e intelectuales de una persona, así como al desarrollo de sus talentos específicos. Es un concepto integrador, que supone el conocimiento de la persona acerca de sí misma y de su unicidad, el planteamiento de metas personales, el reconocimiento de los propios talentos y la formulación de objetivos coherentes con su sistema de valores. En este sentido, el desarrollo personal está íntimamente ligado al concepto de formación afectiva y valórica y al de inteligencia emocional. (Aron y Milicic 2004, p. 17)

A este concepto se le puede agregar, que se encuentra marcado por la cultura, clase social, género, etapa de vida, condición socioeconómica, entre otras variables importantes. Se desprende de lo anterior, que el desarrollo personal se divide en varias áreas, tales como: -socio-afectiva, cognoscitiva y psicomotora. En el área socio-afectiva-, se circunscribe la dimensión vocacional que, a su vez, incluye los intereses, aptitudes, valores, toma de decisiones, elección vocacional, proyecto de vida y metas. En el ámbito emocional, se toman en cuenta la identidad, autoconcepto, autoimagen, autonomía, autoestima, iniciativa, creatividad y flexibilidad. En la parte social, las habilidades para la comunicación interpersonal, solidaridad, cooperación, respeto, tolerancia, organización y participación. Por último en el aspecto ético-espiritual; los valores personales, morales, laborales, familiares y espirituales, (Campos et al., 2002).

Es importante destacar, que existen también diferencias genéricas en torno a la conformación del desarrollo personal para hombres y para mujeres. Delgadillo (1996) explica que entre las paradojas del sistema genérico patriarcal, el hombre es considerado "ser independiente", este siempre depende de los cuidados maternos de la mujer, sea esta madre, hermana, hija, esposa, compañera. La mujer por el contrario, considerada "dependiente" es la encargada de prodigar cuidado y atención a la familia. Por esto motivo, 
aunque tenga la posibilidad de un desarrollo más allá de las fronteras de la esfera doméstica, continúa ligada a ésta.

En síntesis, el desarrollo personal, es un proceso de construcción continuo que toda persona experimenta, en el cual la identidad genérica, juega un papel importante para la conformación personal, ya que afecta todas las áreas de vida. El desarrollo personal está, igualmente muy relacionado, con fuerzas constructivas internas poseídas por cada persona de ahí que encierra el crecimiento emocional y social. Para enriquecerse, requiere, especialmente, de la habilidad de la comunicación.

\subsection{Desarrollo Profesional de la mujer en la docencia}

El ámbito laboral resulta muy importante en la vida adulta, este ocupa una porción significativa de las tareas diarias, constituye parte central de los ámbitos de acción para el ejercicio de los conocimientos propios del trabajo, así como para la práctica de distintas habilidades y capacidades personales. De acuerdo con los intereses del presente artículo, es importante retomar la labor profesional y caracterizar a las maestras de educación primaria como profesionales de la docencia.

El desarrollo profesional es descrito por Alfaro, Montoya, Rodríguez, Solano y Solano (2003) como un proceso amplio y flexible, compuesto por una serie de fases de maduración vividas en el transcurso de la vida profesional y que se enfrentan de manera personal. Según este aporte, esa formación de la persona docente se concibe como un proceso continuo, sistemático y organizado, que abarca la totalidad de la carrera docente.

El proceso de enseñanza requiere que la población profesional docente, ejecute toda una gama de habilidades de diversa índole, puesto que las exigencias son altas en su labor diaria, lo que a su vez implica que experimente distintas etapas. Cornejo (1998) identifica tres etapas relevantes en el desarrollo profesional:

- Formación básica y socialización profesional (preparación en la Universidad): Aquí se produce una primera transformación y eventuales cambios de las actividades, valores y funciones que la población estudiantil como futuras o futuros profesionales en educación atribuye a la profesión docente y la generación de determinados hábitos que influirán en su ejercicio docente posterior.

- Inducción profesional y socialización en la práctica (tres primeros años en el ejercicio profesional): Corresponde a los primeros años del ejercicio docente, en que la persona profesional debutante, aún en proceso formativo, tendrá ciertas características 
especiales.

- Perfeccionamiento (mejoramiento a lo largo de toda la vida como docente): En esta etapa, relucirán actividades propias de la formación permanente, tales como reflexión, comprensión e intervención a partir de la propia práctica, intercambio de la experiencia y actualización constante.

La última etapa, correspondiente al perfeccionamiento, concuerda con las docentes participantes, debido a que se ubican en ésta. Conocer las características de estas etapas permite comprender la forma en que las docentes utilizan sus distintas habilidades y conocimientos, y, como éstos les favorecen en su desarrollo personal y por ende, profesional.

Es importante retomar la condición femenina profesional en la sociedad actual, caracterizada por una parte, por cambios hacia relaciones cada vez más equitativas entre hombres y mujeres en comparación con las sociedades antiguas; por otra parte, ese cambio positivo entre las personas de distintos géneros, etnias y clases sociales, depende en buena medida de las oportunidades que el mismo entorno social ofrece para replantear y construir una nueva convivencia, por ello se hace necesario destacar algunos aspectos enlazados con la mujer y la docencia como ámbito de acción profesional.

En Costa Rica, de acuerdo con Delgadillo (1996), las tendencias sugieren que el hecho de pertenecer a uno u otro género, parece ser una variable fundamental en la escogencia de una carrera profesional universitaria. De esta forma, las mujeres escogerían carreras que prolonguen en el trabajo su rol fundamental, el doméstico; con funciones propias del espacio otorgado a lo femenino desde el patriarcado. Por consiguiente, con tendencia a elegir aquellas que poseen en sí mismas un rol educativo y afectivo, tales como educación, enfermería, psicología, trabajo social, entre otras.

Aunque según lo anterior, a las mujeres se les ha limitado socialmente debido a una división del trabajo basada en el sexo, esta misma división sexual, también, ha favorecido habilidades relacionadas con los sentimientos. La responsabilidad de cuidar a otras personas en las familias, exige una atención, dedicación, interés y una conciencia social respecto del bienestar de otra persona. Como gestoras del ámbito doméstico, personal y emocional, las mujeres conservan una gran capacidad para el contacto humano (Orbach y Eichenbaum, 1989).

No obstante, al estar socializadas en una cultura patriarcal, también suelen asumir patrones masculinos negativos avalados por la sociedad para conseguir éxito. Igualmente, 
suelen hacer mal uso de las anteriores habilidades, lo cual se traduce en consecuencias nocivas para su propio desarrollo personal, al entrar en contradicción con ciertas habilidades que se poseen. Lo anterior, es necesario retomar para comprender la condición de las mujeres profesionales en la docencia, inmersas en un ambiente social educativo, que les exige responder a diversas tareas tanto en el ámbito personal como profesional y para los cuales, la Orientación facilita y acompaña en los distintos procesos del desarrollo humano, como el autoconocimiento, la identidad, el conocimiento del medio, la toma de decisiones y proyecto de vida.

\subsection{Orientación a la mujer adulta}

La Orientación como disciplina dirigida a favorecer procesos de crecimiento a todas las personas durante el ciclo vital, y en este caso, en la etapa adulta, permite construir con las participantes, una nueva perspectiva de afrontar la vida con mayor esperanza, a partir del descubrir, fortalecer o bien, potencializar las habilidades propias, en una relación de responsabilidad compartida entre la persona orientada y el profesional en Orientación.

Como se ha descrito, uno de los propósitos del presente estudio es ofrecer aportes desde la Orientación para propiciar estilos de vida saludables en el ambiente educativo, dirigido a personal docente de educación primaria por erigirse como modelos para niñas y niños, basándose primero en el desarrollo personal, con el propósito de que mediante el desarrollo y confianza de sus propias fortalezas personales, puedan también fortalecer sus relaciones sociales y por ello, laborales, previniendo posibles conflictos o transformando la propia visión de mundo mediante un manejo adecuado de sus habilidades personales y profesionales.

En consideración de Gordillo (1988), la Orientación facilita procesos para lograr el desarrollo personal, en este se acompaña a la persona, de manera que pueda tomar sus propias decisiones con libertad y de acuerdo con su realidad particular. Es importante rescatar, que la persona posibilita su desarrollo personal mediante la influencia de valores de sentido, tales como la libertad, la responsabilidad, la autenticidad, el amor, entre otros. Estos a su vez, son los que suponen un mayor desarrollo personal al haber trascendido la fase del yo individual y que permiten una comprensión del mundo que rodea a la persona, enriqueciendo y configurando una imagen de su ser. Gordillo (1998) enfatiza también, que al orientar a una persona adulta, es necesario conocer las crisis normales de la etapa del desarrollo, teoría del desarrollo humano, patrones sociales de aprendizaje y la conducta 
social, sesgo de la propia edad e intereses y necesidades, con el propósito de comprenderla y brindar estrategias acordes con la realidad en que las personas se desarrollan, como en el caso de las mujeres docentes.

Lo anterior, se relaciona con la perspectiva teórica del presente artículo, acorde con el modelo de desarrollo humano contrapuesto al modelo médico o remedial, acercándose a modelos educativos recientes que buscan comprender a las personas y se interesan por desarrollar las potencialidades que cada persona lleva dentro de sí misma (Jaramillo, 1997).

El concepto de Orientación, con base en el modelo de desarrollo humano, busca promover el desarrollo saludable de las personas, mediante el equilibrio entre todas las dimensiones de la vida: física, mental, social, vocacional y espiritual, cada una de ellas influenciada por las otras y por el ambiente. En síntesis, de acuerdo con esta posición, cada persona tiene el control de sí misma, de su propia vida y por lo tanto, posee el poder de cambiar aquellos aspectos que no son confortables con sus vidas; por ejemplo cuando la persona desarrolla respuestas mal adaptadas en cualquiera de las cuatro áreas esenciales del funcionamiento humano, (Hershenson y Power, 1987).

Es importante resaltar que para trabajar con mujeres desde la labor orientadora, se ha de promover en ellas el empoderamiento, el cual tal y como lo aporta León (1997), es un proceso en el que las mujeres incrementan su capacidad de configurar sus propias vidas y su entorno, una evolución en la conciencia de las mujeres sobre sí mismas, en su estatus y en su eficacia en las interacciones sociales. Para poder llegar al empoderamiento, se necesita tomar conciencia de los propios recursos personales, mediante un examen crítico de los paradigmas definidos culturalmente y asimilados por la socialización. Tiene que estar dirigido a romper la cultura del silencio entre las mujeres, como por ejemplo con la dicotomía entre la esfera pública versus privada, de una ideología sostenida por el derecho, la religión y la cultura, para justificar el control de la movilidad, la sexualidad y las capacidades productivas de las mujeres.

Por su parte, Rogers (1989) aporta que para el trabajo con personas adultas, en grupos de Orientación, es necesario trabajar elementos que enriquezcan la comunicación, como la habilidad sensible de escuchar, la autenticidad, que a su vez estimula la autenticidad de las demás personas y una mayor libertad para dar y recibir amor.

En este sentido, es importante fomentar en las féminas docentes, desde la labor orientadora como mediadora de procesos de crecimiento personal y por ende profesional, una revisión crítica, pero sensible, de sus recursos personales, con el propósito que puedan 
implementarlas en sus vidas y que a su vez, éstas traigan beneficios para las distintas personas con las que ellas se desenvuelven, tomando en cuenta, la necesidad que la persona, se encuentre bien consigo misma y así después pueda proyectarse hacia la sociedad en general.

\section{Acercamiento Metodológicos}

La naturaleza de las interrogantes planteadas, privilegió la elección del paradigma naturalista, el cual presentó valores fundamentales para el desarrollo de la investigación, ubicándola al servicio de las personas.

Se utilizó un proceso de investigación cualitativo, que, para Tamayo (2002), es de naturaleza progresiva y obedece a una modalidad de diseño semi-estructurado y flexible, esto quiere decir que los supuestos no son fijos a lo largo del proceso de investigación, sino que se trabaja con un enfoque heurístico o generativo, debido a que cada descubrimiento en relación con este se convierte en el punto de partida de un nuevo ciclo investigativo inmerso en un mismo proceso de investigación.

Se optó por la postura teórica de Orientación del desarrollo humano, que englobó de forma consistente y coherente tanto el propio pensar, sentir y actuar de la persona investigadora, como de los propósitos del estudio. Esta postura se enfatizó en las fortalezas y no en las debilidades de las personas, se dirigió hacia las necesidades de desarrollo más que a las necesidades remediales; se asumió una relación simétrica entre la persona investigadora y las personas participantes y concibió, como logros apropiados en la Orientación, que las personas orientadas lograran expresar sus percepciones sobre el propio desarrollo personal y profesional, con el propósito de mejorar en esas áreas e incrementar su dominio personal en las tareas de vida.

Para acercarse a las informantes, se optó por aproximarse con el método fenomenológico y después profundizar con las entrevistas, debido a la profundidad de este método, por esto se refiere a una aproximación al método. La persona investigadora adscrita a la fenomenología, encierra una profunda necesidad de comprender la naturaleza humana inmersa en el mundo social. Utiliza métodos que también responden a un acercamiento ético que trata de rescatar de forma científica la subjetividad para la comprender las intenciones humanas. El acercarse con la fenomenología, permitió no centrarse solamente en el contexto como con otros métodos, como el etnográfico, sino en las personas participantes, sino más 
bien, comprender cómo el contexto educativo les ha aportado o no, en ese desarrollo personal y laboral.

Se utilizó la técnica de la entrevista en profundidad para enfocarse en aquellas cualidades y habilidades señaladas por las participantes como más significativas, que pudieran favorecer el propio desarrollo personal y profesional de la persona docente. Posteriormente se realizó una devolución de la información mediante el grupo de discusión como técnica que permitió validar los datos obtenidos con la entrevista. Con el propósito de organizar el proceso de investigación, se siguieron diferentes momentos. Se describen brevemente a continuación:

\subsection{Primer momento: selección de las personas participantes y descripción del contexto}

Se valora el apoyo institucional y el interés personal de las personas participantes del estudio. Se elige una institución de educación formal de tipo semi privada subvencionada por el Estado, para realizar el estudio, debido a la anuencia tanto de la institución como de las informantes, la conveniencia y el interés de la propia investigadora.

Se eligieron cuatro maestras que laboran en el primer ciclo de educación primaria, que imparten asignaturas básicas dentro de esta institución, por ser las que poseen mayor experiencia profesional de acuerdo con el tiempo servido en la docencia, con un mínimo de cuatro años. Este requisito se fundamenta en el aporte de Cornejo (1998) sobre las etapas del desarrollo profesional, que para el caso de las maestras participantes estarían ubicadas, en la etapa de "Perfeccionamiento" (mejoramiento a lo largo de toda la vida como docente). Se caracteriza esta etapa, por actitudes de reflexión, comprensión e intervención a partir de la propia práctica, intercambio de la experiencia y actualización constante, lo que calzó perfectamente con la realidad profesional de estas mujeres y ofreció elementos importantes para el estudio, en cuanto a sus experiencias profesionales.

Se justifica esta cantidad de participantes, debido a que el número de casos en la investigación cualitativa carece relativamente de importancia, sino que se enfoca en el potencial de cada caso como medio para la comprensión de las preguntas formuladas en el presente estudio (Taylor y Bogdan 1996).

Fue del interés investigativo que fueran maestras del primer ciclo, por la relación y el contacto con la población infantil, sus madres, padres y otros colegas docentes y las mismas autoridades institucionales, que podrían implicar atender una serie de demandas de índoles 
social, emocional y cognitivo, sin descuidar el propio desarrollo personal. Las edades de las mujeres participantes oscilaron entre los treinta y cuatro y cuarenta años. Sus lugares de residencia están ubicados en zona urbana, cercanas a Guadalupe y Tibás; pertenecen a sectores medios de la estratificación socioeconómica. Todas ellas con título de bachillerato universitario en Educación General Básica para I y II ciclos. En cuanto al estado civil, una de ellas es soltera, una divorciada y dos casadas. Para hacer referencia a las mujeres participantes, se hizo uso del anonimato. Se utilizó la definición de Participante \#..." para garantizar la confidencialidad de la identidad y de la información suministrada.

\subsection{Segundo momento: Descripción de la entrevista en profundidad}

Se eligió la entrevista en profundidad, por la ventaja tanto para los propósitos del estudio como por la realidad de las maestras participantes. Sus múltiples tareas, requirieron de espacios flexibles que les permitiesen sentirse cómodas y a la vez facilitar la recolección de la información, de manera que las participantes se pudieron expresar libremente en cuanto a sus pensamientos y sentimientos. La investigadora realizó los ajustes necesarios a la realidad de ellas y no al contrario.

La entrevista en profundidad de acuerdo con Valles (1997) es una de las técnicas preferidas en la investigación naturalista que adopta la forma de un diálogo o una interacción. Posee la ventaja de intercambiar información y establecer una relación de apoyo de manera que ambas partes se favorecen.

Se hizo uso de la entrevista basada en un guión que permitió libertad, ordenar y formular preguntas a lo largo del encuentro. Según López (2002) en las entrevistas en profundidad no debe faltar una planificación por parte de la persona investigadora, que incluya el guión; que es un sencillo escrito en el que se diseña el proceso esperado de la conversación, mediante la reseña de los hitos que deben marcar la secuencia temática. Al realizar la entrevista cara a cara, proporcionó mayor intimidad entre investigadora y persona entrevistada. Brindó un espacio de clarificación de sus propias situaciones de vida que para efectos del estudio con las maestras participantes, fue oportuno y acorde con el tema. Se hicieron ocho acercamientos con las informantes con un promedio de tres horas por conversación. 


\subsection{Tercer momento: Ejes temáticos del guión por desarrollar en la entrevista en profundidad}

Para seleccionar los ejes temáticos, se revisó la información referente al tema de investigación de interés que se encontró existe en Costa Rica, así como una consulta con personas expertas en el campo, que permitió realimentación y enriqueció ese momento investigativo. Posterior a esto, se revisaron los propósitos del estudio para que fueran compatibles con las interrogantes formuladas, los intereses de la investigadora, el contexto, el modelo de Orientación y del paradigma de investigación naturalista.

Los ejes temáticos fueron sugerentes y en absoluto se tomaron como únicos o rígidos, debido a que estaría en contra de los principios mismos de la investigación cualitativa. Para efectos de realizar las entrevistas en profundidad, se elaboraron los siguientes temas por explorar relacionados con el desarrollo personal: percepción del propio desarrollo personal, vivencia del desarrollo personal, cualidades más importantes para ellas en el ámbito personal, su condición de mujer en el ámbito personal, recomendaciones para el desarrollo personal de maestras. Los ejes temáticos del desarrollo profesional fueron: percepción del propio desarrollo profesional, vivencia del desarrollo profesional, habilidades más importantes para ellas en el ámbito profesional educativo, su condición de mujer en el ámbito profesional educativo y recomendaciones para el desarrollo profesional de maestras.

\subsection{Cuarto momento: tipo de análisis y validación de la información}

Se utilizó el análisis en progreso, por el tipo de información recolectada y por respeto a la confidencialidad de las participantes. Este permitió la recolección de los datos y análisis de forma paralela, de manera que se pudo desarrollar una mejor comprensión de las personas que se estudiaron.

De acuerdo con Taylor y Bogdan (1996) implica ciertas etapas diferenciadas, las que se aplicaron de la siguiente forma:

- La primera es una fase de descubrimiento en progreso. Se identificaron los temas y se desarrollaron conceptos y proposiciones. Se leyeron y releyeron las transcripciones de las entrevistas en profundidad, se volvió al campo para ampliar y aclarar la información. Se hizo una primera organización de los datos más importantes. Fue un ir y venir, que permite la familiarización con los datos, para empezar a ordenarlos.

- La segunda fase que se produce cuando los datos ya se han recogido. Se inició la codificación de los datos y se refinó la comprensión del tema en estudio, se elaboró un 
cuadro con categorías y subcategorías, que incluye, expresiones de las participantes que ejemplifican el contenido. Una vez realizada cada entrevista, se transcribe completa, se ordena su contenido según las expresiones de las participantes en categorías y subcategorías, las que se estructuran según los ejes temáticos y las preguntas de investigación. La creación de las categorías y subcategorías implicó una clasificación de temas semejantes, diferentes, contradicciones, pero también puntos de confluencia; se usaron diferentes colores para elaborar esa clasificación y poder clarificar.

- En la fase final, la investigadora relativizó los descubrimientos, es decir, comprender los datos en el contexto en que fueron recogidos. En esta parte, se utilizó la teoría respecto con los resultados y la elaboración de la investigadora, se identificaron los alcances de las preguntas de investigación. Se incluyó en esta parte la validación de la información.

\subsection{Validación}

En la fase final del análisis de la relativización de los descubrimientos, se incluyó la validación de la información. Se hizo uso de la triangulación, que se construyó con las expresiones de las participantes en las entrevistas en profundidad y en el grupo de discusión al devolver la información recopilada, las aproximaciones teóricas e interpretación crítica que la investigadora hace de todo lo anterior.

No se utilizó un programa informático de análisis de datos, sino que lo realizó de forma manual la investigadora del presente estudio. Después de realizadas las entrevistas en profundidad, se hizo uso de la técnica del grupo de discusión, con el total de maestras participantes; que permitió aclarar dudas y ampliar la información obtenida. Según Delgado y Gutiérrez (1998) el grupo de discusión es un tipo de entrevista grupal, en la cual el discurso social se encuentra distribuido en lo social mismo, en ese proceso el discurso reordena para el grupo y el grupo a su vez, ordena según criterios de pertenencia.

Se discutió con las participantes los hallazgos más importantes referentes a los propósitos del estudio, que permitieron validar la información obtenida, es decir, se aclararon y ampliaron aspectos que no fueron abordados en las entrevistas en profundidad respecto con las interrogantes de interés.

La dinámica del grupo de discusión, se hizo siguiendo los siguientes aspectos que aportan Delgado y Gutiérrez (1998): 
- Para la ejecución del grupo de discusión, se utilizó un aula del centro educativo al que pertenecen las maestras participantes del estudio y se llevó a cabo el día negociado con ellas, en el horario establecido.

- El tema se enunció de forma general. Se delimitó por un lado el ámbito de discusión, pero se dejó abierto por otro lado, la discusión. Para este caso, el tema se formuló en torno a posibles aspectos por ampliar respecto de las preguntas del estudio y para corroborar la información obtenida.

- El discurso se grabó mediante cintas de audio, respetando la confidencialidad y familiaridad de las participantes al no utilizar otros medios. La facilitadora tomó notas cuando lo consideró necesario y señaló puntos clave de consenso en el discurso.

- La técnica se explicó de forma concisa, sin complicar a las participantes, no se implicó al grupo en exceso en sus razones o procedimientos.

- La investigadora facilitó la discusión, de manera que no se divagara por otros caminos ajenos al encuadre.

- La investigadora, con sus intervenciones hizo enunciados tratando de tener especial cuidado con las interpretaciones.

- Se les agradeció al grupo tanto al iniciar la reunión, como al terminar; recalcando la importancia de sus opiniones para el buen curso de la investigación. Al finalizar la discusión, se compartió un refrigerio con las participantes.

Esta estrategia permitió por un lado, proteger los resultados obtenidos con el estudio, mediante la combinación de distintos métodos o fuentes de datos, para confrontar y someter a control recíprocos las narraciones de las informantes (Taylor y Bogdan, 1996). Y por otro lado, facilitó que las participantes compartieran sus pensamientos y sentimientos respecto al tema de forma grupal, como una manera de complementar su desarrollo personal, mostrándoles el uso que se hizo de la información, recibir recomendaciones, obtener información adicional y la posibilidad de revisar lo ya existente para modificar aquello que lo requirió.

\section{Análisis}

\subsection{Relativización de los descubrimientos}

Esta sección corresponde a la fase de análisis, que como se explicó anteriormente se elabora mediante una relativización de los descubrimientos. Se presenta el alcance de las 
preguntas de investigación en relación con las maestras participantes del estudio, las aproximaciones teóricas, los hallazgos importantes, así como los aportes de las participantes emitidos durante la devolución de la información, este último proceso con el propósito de realizar la validación de los datos.

La primera pregunta que se planteó fue: ¿Qué concepto personal y profesional de sí mismas, poseen un grupo de maestras de educación primaria? De esta interrogante surge la primera categoría que se titula "Condición de mujer en el ámbito personal" y corresponde a la percepción que tienen de sí mismas como personas, las mujeres adultas participantes.

Las participantes se definen personalmente como mujeres analíticas, que tienden a pensar y ejecutar las metas que se han propuesto, mediante la responsabilidad que las caracteriza, caracterización que rescata elementos similares al concepto de desarrollo personal, descrito por Aron y Milicic (2004), en cuanto al planteamiento y ejecución de metas personales. $Y$ por otra parte, se evidencia necesidad de fortalecer ese desarrollo personal, ya que se caracterizan como personas reservadas en cuanto a expresión de sus pensamientos y sentimientos, siendo conscientes de que los poseen, pero con una tendencia a guardarlos debido a sus propias experiencias pasadas y presentes, que al parecer las han motivado a no comunicarlos abiertamente. Esto podría denotar inseguridad por parte de ellas y una necesidad hasta cierto punto de ser aceptadas por las personas, pero con el temor latente de que al expresarse, se reconozcan en ellas características que les ocasionen rechazo, al ser aspectos posiblemente no integrados o reconocidos de sí mismas.

Las mujeres docentes comparten la existencia de cualidades que según sus percepciones, son muy propias de las mujeres; tales como las relacionadas con el servir a las demás personas, que son estipuladas desde la cultura patriarcal y que las ponen en práctica en sus labores docentes, mediante el cuidado hacia quienes las rodean, implementando la comprensión, el cariño y la sensibilidad, visualizándose todas como madres, pese a que esta condición solo la experimenta una de ellas. Además, esto podría denotar también una caracterización estereotipada hacia la figura materna, que de acuerdo con sus constructos personales, ha de manifestarse cariñosa, sensible y siempre dispuesta para atender las necesidades de quienes conviven con ella. La imagen de madre estereotipada socialmente, se relaciona con el aporte de León (1997) y Delgadillo (1996); quienes explican que como la mujer es la que pare las hijas y los hijos, ella debe ser la encargada de estas personas y del hogar, se le ve como madre o como seductora, esta 
última es la mala mujer, símbolo de destrucción y esterilidad, en cambio, la madre es la venerable, la abnegada y la dispuesta al sacrificio.

Las cualidades que ellas señalan como femeninas afirman poseerlas y tenerlas muy desarrolladas. Dar, servir y proteger a quienes formen parte de sus vidas, es una constante importante, de acuerdo con las descripciones que de sí mismas elaboran, lo que denota estereotipos de género que indican un comportamiento y formas de subjetividad determinadas dependiendo de si se posee un cuerpo sexuado femenino o masculino.

“...Cualidades femeninas verdad, que es ser uno maternal, ser comprensiva, generalmente las mujeres son mas emocionales, menos se dejan llevar por la razón, hay que comprender los sentimientos de los demás, todo eso (...) yo siento que las cualidades propias de una mujer yo las tengo muy desarrolladas". Participante \#2

Podría explicarse también, desde esta perspectiva que al centrarse en las necesidades y mundos de las demás personas, les quede poca energía para redirigirla hacia sus propias vivencias, haciendo difícil un crecimiento personal de la identidad y a su vez, la expresión de sus propios sentimientos y pensamientos que denoten ese quiénes son, es decir, especialistas en atender las necesidades de otras y otros, pero con dificultades para identificar y por tanto atender las propias. Lo anterior se relaciona con el aporte de Bustamante (1997) al afirmar que la condición de la mujer a lo largo de la historia, puede ser descrita desde la palabra crisis, debido a que se caracteriza por el cambio, el conflicto y la tensión. La crisis ha sido la compañera inseparable de la mujer en la búsqueda de su propia identidad, debido a que la evolución de su condición ha sido una constante lucha contra estereotipos y prejuicios sociales que la han sojuzgado.

Resulta interesante destacar, que existe conciencia en una de las participantes, al criticar la condición estereotipada de ser mujer en la sociedad costarricense, tal y como lo ha percibido de acuerdo con su propia historia de vida, que obliga a que ellas mantengan una posición de subordinación ante el cónyuge en contraposición a una relación más equitativa entre hombre y mujer, lo cual desde su perspectiva, al implementar un comportamiento distinto, lleva al fracaso en la relación de pareja.

"La situación social te dice que la mujer sigue al hombre y yo luché porque él me siguiera a mí (risa), entonces ahí hubo un desbalance, bueno al menos aunque no me siguiera a mí, hubiera un equilibrio como es lo que debe ser y como no se logró por el machismo y todo ese asunto, entonces fracasé..." Participante \#4 
Es interesante percibir que para las participantes es importante mantener una relación de pareja, la maternidad o funciones asociadas a ellas, para lograr la realización como mujeres desde la condición personal, debido a que si no es de esta forma, existen sanciones sociales por las cuales es mejor elegir ciertos sustitutos aceptados socialmente como las anteriores funciones, lo que podría responder a las necesidades manifiestas por estas mujeres.

La realización personal de estas mujeres se asocia con características estipuladas socialmente que deben poseer las mujeres, haciendo una comparación con las supuestas tareas señaladas como propias para las mujeres y la forma en que estas están presentes o no en sus vidas. Se evidencia en ellas, ante estas tareas una cierta resignación al asumirlas, pero también insatisfacción si no logran cumplir con el mandato de ser madres o esposas y ser las especialistas en el mundo privado, más que en el mundo público fuera del hogar, aunque tengan logros en el mundo profesional, son opacados por un mandato social de perfección, lo que concuerda con lo señalado por Bustamante (1997).

"Me siento orgullosa de mí misma, de mis metas, de donde he llegado, de donde he logrado colocarme, de las cosas que he hecho como mamá me siento súper orgullosa, súper satisfecha, como mujer no mucho... porque no he logrado entablar... no logré establecer una relación de pareja, un hogar firme sí, pero una relación de pareja buena no la tuve..." Participante \#4

Pese a que estas mujeres señalan logros personales, no son reconocidos en su totalidad para tener realización personal. Se percibe como consecuencia, que las docentes manifiestan una división de sí mismas, es decir, por una parte mujeres con sus vidas privadas y por otro lado mujeres profesionales docentes, quienes a pesar de sus logros no logran integrar todo ello para realizar una evaluación positiva de sí mismas. Aspecto interesante de resaltar, acorde con el aporte de León (1997) quien señala que la dicotomía que favorece la sociedad patriarcal de esfera pública versus esfera privada, fragmenta la vida de las personas, en especial de las mujeres, quienes históricamente han sido conformadas para funcionar y transmitir la cultura de desde esa visión, lo que les ocasiona sentimientos y pensamientos de inferioridad, que por supuesto repercute en las distintas esferas en que se desenvuelvan.

En síntesis, la condición de ser mujer en el ámbito personal de las participantes, se ve impregnada de estereotipos de género, tales como estar al servicio siempre de las demás 
personas por encima de sus propias necesidades, cuestionamientos y contradicciones, lo cual lleva a pensar en la construcción permanente de estas mujeres respecto de sus propias identidades y los momentos críticos que enfrentan en la implementación de decisiones que les permitan transformar sus vidas. Este último aspecto, denota fortalezas internas, que pueden ser aprovechas para potencializarlas tanto desde el modelo de desarrollo de fortalezas de Orientación descrito por Jaramillo (1997), como también mediante el empoderamiento, de acuerdo con León (1997). Pero, también se destacan condicionantes sociales que las juzgan, cuando tratan de moverse de ciertas tareas impuestas e implementar distintas habilidades que quizás no son señaladas desde el patriarcado como propias de las mujeres, pero que por la presión social son asumidas por ellas, como una características natural y por tanto no cuestionable.

La segunda interrogante que se planteó fue: ¿Qué aspectos considera el grupo de maestras se requieren para favorecer el propio desarrollo personal y profesional?

De esta surge una segunda categoría se titula "Condición de mujer en el ámbito profesional", corresponde a la percepción que tienen de sí mismas como mujeres profesionales de la docencia, las adultas participantes.

Un hallazgo importante es que se señala por una parte, que las mujeres profesionales de la docencia, poseen ciertas habilidades muy propias de ellas y una especie de instinto para deducir y comprender fácilmente los sentimientos de las demás personas, en especial de la población infantil. Pero, por otra parte, al acercarse a la experiencia personal, se distingue como recomendación no involucrarse afectivamente con niñas y niñas, debido a que esto ocasiona dificultades de distanciamiento emocional posterior entre la docente y el grupo de estudiantes con las consecuencias negativas para ambos, es decir, podría reflejarse un discurso social que es necesario mantener, pero por otra parte, una identificación de posibles descontentos ante esos mandatos personales reflejados en el propio desarrollo personal y profesional.

"Bueno sobre todo, un.... digamos, yo lo que... es que son como cosas que uno tiene, que uno nace con eso como mujer, verdad? Porque no es porque uno las haya cultivado, ni nada por el estilo, lo principal que yo veo que tengo, es una habilidad grande en eso, para entender a los demás, para como saber que piensan, o... un don o no sé qué será... simplemente yo veo que soy así..." Participante \#2 
"Mi relación con ellos sí es un poco distante... porque no puedo apegarme tanto a los chiquillos, porque el bombazo a final de año es muy grande, ya lo experimenté un año y fue fatal... y tampoco uno tiene el tiempo... como para acercarse un poco más a ellos, porque yo siento que no es saludable para ninguno de los dos, ni para uno ni para ellos, porque después viene el cambio de docente y hay muchos problemas, trato de... de diay de ofrecerles mi ayuda en todo lo que yo pueda... pero siempre sí... manteniendo distancia" Participante \#3

Esta posición es compartida por el resto de participantes, al enfatizar que resulta saludable en el ámbito emocional, evitar contacto cercano con niñas y niñas, como anticipación de posibles consecuencias emocionales negativas ante la separación. Pero por otro lado y en oposición a lo anterior, se destaca en algunas de las docentes participantes, una mejor relación con niñas y niños, a partir de la identificación de ciertas características personales que comparten con la población infantil, lo que les facilita la interacción con ellas y ellos, a diferencia que con las personas adultas.

"Bueno, pues que no dejé de ser niña, yo siento que para mí es muy fácil hacerme una niña como ellos y ver las cosas como ellos las ven y bromear... ponerme a jugar con ellos y todas esas cosas a mi no me cuesta, entonces, no sé, es como esa.. esa, facilidad que tengo." Participante \#2

"Sí con ellos tengo que ser un poquito más hábil socialmente (...)Tal vez yo digo que esa forma de tratar de usar un poquito la psicología inversa, verdad?... porque con los papás ya te digo... hay papás que yo sé que me estiman, que me aprecian como soy... pero hay otros que no me soportan... verdad? seguro desean como matarme y cortarme la yugular, pero no me afecta y yo soy feliz con los chiquillos, dentro del aula... Participante \#4

Se podría interpretar que la profesión docente les proporciona a estas mujeres un espacio de protección al relacionarse con la población infantil, para la cual se sienten capacitadas, debido a que no reciben de ellas y ellos críticas que les afecten, es decir, no son juzgadas. No así de las personas adultas, que es con quienes expresan ciertas resistencias para relacionarse, al manifestar desconfianza, temor y hasta rechazo; que podrían ser percibidas por estas mujeres, como posibles jueces de sus debilidades, pero en 
realidad, podría estar detrás de sí mismas, una persona no aceptada, primero por ellas mismas y después por las demás, que constantemente hace que se juzguen a sí mismas y que no permite que se comporten de forma espontánea, disfrutando y aprendiendo de las mujeres que realmente son, con debilidades por superar, pero también con muchas fortalezas importantes.

Por otra parte, señalan a la profesión docente como muy femenina y que los hombres que la desempeñen deben poseer igualmente cualidades que desde la cultura patriarcal, se atribuyen a las mujeres, como la comprensión y las relacionadas con la maternidad. Hacen una división y reconocen que ciertas características les pertenecen solo a las mujeres y existen otras, que son competencia de los varones. Se denota aceptación y satisfacción de las participantes porque sea un campo exclusivamente de las mujeres y hasta rechazo y desconfianza hacia los hombres que ejercen la docencia; resaltan ventajas de su condición femenina al relacionarse con madres y padres de familia, a diferencia de sus colegas docentes varones. Esta crítica que se evidencia en sus comentarios, fue compartido por todas las informantes.

"A un hombre siempre le tienen la muralla de que es hombre, puede... suceder algo más como sea... con todos los mitos que han sucedido, entonces esos pobres ¡diay! los tiene como tachados, no digo que con mujeres no se ha dado, verdad? Pero es menos...el susto para los padres de familia que puede suceder algo más... para el hombre es más difícil en el sentido de acercamiento a los niños... Participante \#1

De acuerdo con Delgadillo (1996) en Costa Rica las tendencias sociales sugieren que el hecho de pertenecer a uno y otro género, parece ser una variable fundamental en la escogencia de una carrera profesional universitaria. De esta forma las mujeres escogerían carreras que prolonguen en el trabajo su rol fundamental, el doméstico, con una tendencia a elegir profesiones que tengan en sí mismas un papel educativo y afectivo, tales como las del área educativa, o psicología, enfermería o trabajo social entre otras.

Se destacan algunos prejuicios o diferencias latentes en la relación laboral con los varones docentes. Señalan que los hombres son competitivos y que existe mucha rivalidad cuando están presentes, que incluso tienden a menos preciar el trabajo que realizan las mujeres. De acuerdo con sus experiencias, los compañeros varones que laboran en secundaria que constituyen mayoría, manifiestan indiferencia ante los aportes que se hacen por parte de las docentes de primaria, aunque ellas son mayoría en primaria. 
"Los hombres tienden a ver a la mujer por debajo de ellos, tiene como un complejo de superioridad. Participante \#3

"Aquí inclusive se da que muchas veces en secundaria, tratan de pasar por alto lo que proponemos las de primaria y en secundaria trabajan más hombres que mujeres" Participante \#1

En esta comparación entre hombres y mujeres docentes que denota ciertos mitos, también se visualiza el deseo de poseer la practicidad, cualidad que le atribuyen al género masculino, así como el hecho de no involucrarse emocionalmente con las personas con quienes trabajan, lo que les trae más beneficios, pese a que ellas describen por otro lado, lo aparentemente deseable que son las cualidades maternales trasladada a su labor.

"Los hombres son más tranquilos, más pragmáticos, son como más... o sea, ellos dicen: -yo hago lo que tengo que hacer, si no responden, no es mi problema-; jellos son felices!, en cambio uno se afecta más, uno se involucra más emocionalmente, el hombre maestro no, jel hombre va a lo de él y ya está!" Participante \#4

De acuerdo con los aportes anteriores, se rescata que la condición de la mujer en el ámbito profesional docente, se evidencia muy similar desde la perspectiva personal, caracterizada por demandas sociales desde el género que las obligan a actuar de determinadas formas si son mujeres u hombres, incluso, para la labor profesional que realizan, señalan ventajas al ejercer la docencia porque de acuerdo con sus percepciones, es muy femenina. En este punto se encuentra relación con el aporte de Orbach y Eichenbaum (1989) quienes afirman que las mujeres al ser designadas como gestoras y especialistas en lo doméstico del mundo privado, personal y emocional, conservan una gran capacidad para el contacto humano y son capaces de desarrollar cualidades y habilidades que les proporciona ventajas. Pero por otra parte, se vuelve un mandato social incuestionable presente en el desarrollo profesional de estas mujeres docentes, con procesos importantes para su conformación personal, a los cuales la Orientación aporta reflexión, análisis y nuevas alternativas ante las situaciones que se experimentan.

La tercera interrogante planteada fue: ¿Qué aportes puede brindar la Orientación para el desarrollo personal y profesional de maestras de educación primaria? 
En este punto, las maestras se enfocan en la necesidad de favorecer un mayor conocimiento de sus fortalezas, de ser escuchadas y de obtener espacios para relacionarse con las personas. Sobresale que la labor que deberían realizar profesionales en Orientación, debe rescatar el trabajo coordinado con las mujeres docentes, debido a que en ocasiones, estas personas especialistas en el cambo, no toman en cuenta sus recomendaciones y optan por asumir una posición autoritaria al tratar de solucionar un conflicto de forma unilateral.

"El hecho también de poder trabajar en conjunto con los padres de familia, cosa que no se hace, por ejemplo, Orientación por aquí y yo soy Orientación, usted es la maestra, pero yo soy Orientación, no hablo solo de esta institución, pero como que el orientador se hace... como que tiende a hacerse el problema como propio, se le olvidó que fue el docente el que refirió el problema." Participante \#3

Aspectos compatibles con tomar en cuenta la cooperación de todos los agentes educativos (persona profesional en Orientación, docente, equipo directivo, madres y padres, comunidad) con el propósito de ofrecer un servicio integral, que tome en cuenta la comunidad educativa, respetuoso de las funciones y los aportes que cada parte pueda ofrecer, (Gordillo, 1988).

Existe la necesidad, entre las participantes de ser escuchas y validadas, por parte de los distintos miembros educativos y muy importante, la escucha respetuosa de la persona profesional en Orientación, que rescate no solo las debilidades, sino que les haga saber sus fortalezas en la labor diaria que realizan, lo que se dirige nuevamente a la necesidad de no ser juzgadas.

Situación que respalda Rogers (1989) para el trabajo con personas adultas en grupos de Orientación, al señalar un énfasis en elementos que realzan la comunicación, como una habilidad sensible para escuchar, una profunda satisfacción al ser escuchado, habilidad para ser auténtico, que a su vez estimula la autenticidad de las demás personas y una mayor libertad para dar y recibir afecto.

Tanto desde las entrevistas en profundidad como durante la devolución de la información, ellas enfatizan en la importancia de trabajar en Orientación individual para lograr un mayor conocimiento de sí mismas, pero sobre todo la Orientación grupal, ya que les permitiría enriquecerse con la experiencia de otras mujeres docentes, que les brinden ejemplos concretos de cómo enfrentar situaciones de difícil manejo en la parte social, por 
ejemplo al atender a madres y padres de familia o cómo dirigirse a grupos grandes de personas, de manera que puedan superar inseguridades al interrelacionarse y fortalecer otros aspectos positivos de sí mismas en esa interrelación.

"Yo creo que un tanto importante son los talleres, verdad?... o algún tipo de actividad que permita como el intercambio de experiencias... porque a pesar que sea algo que a uno no le ha pasado, no se está exento, entonces yo creo que son cosas muy bonitas. Por ejemplo aquí cuando hacemos algún taller o tenemos algún espacio para compartir y entonces uno se queda a veces como analizando todo eso y dice: -bueno que dicha que lo hicimos- porque a veces eso sirve para limar alguna aspereza que haya quedado por ahí y eso te ayuda también a crecer... cuando no se dan esos espacios, uno se queda con muchas cosas ahí adentro... por el corre, corre o por la falta de valor, no las afronta y no las trata de resolver, pero si te dan esos espacios, por lo menos se puedan dar pasos importantes." Participante \#3

"Qué curioso, porque no me imaginaba que mis compañeras, también se sintieran así como inseguras, yo pensé que sólo yo era así..." Participante \#2

Esto se relaciona con lo aportado por Gordillo (1984) quien afirma que la Orientación de forma general es entendida como la ayuda ofrecida para lograr el desarrollo personal, en la que existe la intención de promover un mayor crecimiento, desarrollo, madurez y capacidad de afrontar la vida, dirigida a las personas de forma individual o grupal.

Para hablar de las dificultades que sienten las mujeres y de la percepción de la otra persona, es fundamental emplear un lenguaje sensible que pueda ayudar tanto a la mujer orientada en este caso, como a la facilitadora del proceso, apuesto que ambas mujeres, comparten esa realidad que las une históricamente, para lo cual es importante encontrar formas de comunicarse que no suscite culpabilidad en la otra, ni imponga exigencias imposibles de satisfacer por su misma esencia. Tal y como lo apunta León (1998), se debe facilitar el empoderamiento, muy relacionado también con el enfoque de desarrollo desde la Orientación según Jaramillo (1997), aspectos que deben contemplarse en los procesos de Orientación en los cuales se trabaje con mujeres.

Desde los hallazgos encontrados en el presente estudio, la Orientación debe propiciar espacios para el desarrollo personal y profesional, ya que como lo confirman las expresiones de las docentes, existe una interrelación entre el desarrollo personal y el desarrollo 
profesional y por lo tanto, se hace necesario un traslado importante de lo que se es como persona y como profesional. Es importante, destacar según la perspectiva de Orbach y Eichenbaum (1989), que para el trabajo con mujeres, es importante un trabajo sensible y que se ajuste a ellas, ya que enfatiza que las mujeres profesionales y en sus relaciones con otras personas, han de tener la valentía de examinar dificultades que surgen entre ellas y abordarlas sin rodeos, pues no son insuperables, poniendo en relieve la capacidad para enriquecerse mutuamente y el sincero cariño que une a cada mujer en una sociedad patriarcal, lo cual le ayudará a progresar en sus relaciones y profesiones.

\section{Conclusiones}

El estudio permitió conocer elementos importantes relacionados con el concepto personal y profesional de un grupo de maestras de educación primaria, así como los aportes que puede brindar la Orientación para ese desarrollo personal y profesional. Si bien se ofrece información que no puede generalizarse para otras personas, sí podría ser utilizada para favorecer el proyecto de vida en las mismas maestras participantes o bien, proporcionar insumos para estudios similares.

Se brinda a continuación lo que se concluye a partir de las interrogantes planteadas, que fueron guía importante en el estudio:

Las mujeres adultas participantes tienden a centrarse en las necesidades y mundos de las demás personas, esto les resta energía para redirigirla hacia sus propias vivencias, haciendo difícil una construcción personal de la identidad. Las maestras se convierten en especialistas en la atención de las necesidades de otras personas, pero con dificultades para identificar y por tanto atender las propias. Por tanto, la condición femenina en el ámbito personal, se percibe impregnada de estereotipos de género, cuestionamientos y contradicciones, esto lleva a pensar en la construcción permanente de estas mujeres respecto a su propia identidad y los momentos críticos que enfrentan en la implementación de decisiones que les permitan transformar sus vidas. Aspecto que denota fortalezas internas, pero también contradicciones sociales que las juzgan, cuando tratan de moverse de ciertas tareas impuestas e implementar otras habilidades que quizás no son señaladas como propias de las mujeres, pero por la presión social, son asumidas de forma natural y por lo tanto no cuestionables.

Es importante también según estas mujeres, darse a sí mismas espacios personales que reflejen sus intereses mediante actividades que las hagan sentirse satisfechas, que les 
permita sencillamente estar con ellas mismas, aspecto interesante, que refleja un contraste ante ese siempre anteponer las necesidades personales por las de las demás personas y que visualiza la importancia de reconocer esos aspectos personales para fortalecer el desarrollo personal y hacerle frente a las múltiples tareas que les demanda su medio.

Las mujeres docentes dentro de su desarrollo personal, señalan poseer ciertas habilidades de tipo social, muy propias de su género y una especie de don para deducir y comprender con más facilidad los sentimientos de las demás personas, especialmente con la población infantil. Pero por otra parte, al acercarse a la experiencia personal, ellas recomiendan no involucrarse afectivamente con niñas y niños, debido a que esto les ocasiona dificultades en el ámbito emocional, al tener que distanciarse posteriormente del grupo de estudiantes. Aspecto que podría reflejar un doble discurso social que es necesario mantener, pero por otra parte, una identificación de inconformidad ante esos mandatos personales, que se reflejan en el propio desarrollo personal y profesional.

Consideran para que fortalecer el desarrollo personal, requieren fortalecer la seguridad en sí mismas, debido a los rasgos de timidez con que se definen en las relaciones sociales, hacer respetar sus propios espacios personales, poder relacionarse con mayor seguridad con madres, padres de familia y con los colegas varones docentes y aprender a poner límites, debido a que esto en ocasiones causa una sobrecarga de funciones, producto también de esa necesidad de sentirse útiles y de ser valoradas por quienes les rodean, pero también por sí mismas, lo que les demanda un mayor autoconocimiento.

Señalan la profesión docente como muy femenina y que por lo tanto los hombres que la desempeñen, deben poseer igualmente cualidades femeninas según la cultura patriarcal, tales como la comprensión y las propias de la maternidad. Posición que resalta que las participantes reconocen cualidades que les pertenecen solamente a las mujeres y otras que les competen únicamente a los varones por una parte y por otra parte, que la maternidad se desempeña de cierta manera ligada a la docencia y que además es innata también en las mujeres.

Las participantes afirman por una parte, sentirse satisfechas con su labor docente y logran identificar el significado que para ellas tiene, el ser maestras, que se enfoca en convertirse en amigas de la población infantil, pero siempre manteniendo cierta distancia, marcando límites en el ámbito emocional y dando prioridad a la enseñanza de valores positivos, porque es como un construir o destruir, dependiendo de la labor que realicen. Pero por otra parte, manifiestan poca claridad ante la elección de la docencia como carrera 
profesional y señalan eventos externos que las motivaron a su escogencia, es decir no existió una posición reflexiva en torno a la elección. Indican motivaciones de tipo social, como de reconocimiento por parte de las personas de la labor que realizan, es decir, se convierten hasta cierto punto en figuras de tipo públicas, así también otras motivaciones importantes como la comodidad en cuanto a horario diurno, que les facilita dedicarse a otras actividades tanto del hogar como de otra índole.

En todos y cada uno de los puntos que se concluyen, la Orientación puede aportar en el desarrollo personal y profesional, es este caso, de mujeres docentes. Es necesario enfocarse en trabajar procesos de Orientación con perspectiva de género, para el autoconocimiento de habilidades, intereses, aptitudes y actitudes tanto en el ámbito personal, como profesional. Sobre este último ámbito, es esencial la reflexión y el fortalecimiento de la elección vocacional, que pese a que se manifiestan satisfechas con la labor docente, es importante clarificar cómo y por qué están en la docencia, esto les podría proporcionar insumos valiosos en sus prácticas docentes, partiendo de la etapa del desarrollo profesional en que se encuentran y del cuestionamiento de mandatos sociales genéricos ya que por ejemplo, como ellas mismas lo identifican, muchas veces se hace un traslado de los aspectos de tipo personal al profesional y viceversa, por lo que esos espacios de reflexión como los que proporciona la disciplina de Orientación, vendría a enriquecer y hasta replantear, algunos elementos de sus proyectos de vida.

Sobresale que la labor que podría realizar una persona profesional en Orientación, de acuerdo con las experiencias de estas mujeres, debe girar en torno a favorecer el conocimiento de sí mismas, la escucha activa y respetuosa, promover espacios para relacionarse con las personas, donde pueden practicar habilidades de tipo social que consideran débiles en sus interacciones. La labor profesional en Orientación con docentes de educación primaria, debe posicionarse desde un enfoque de desarrollo de fortalezas, para rescatar el trabajo coordinado con las mujeres docentes, validar los aportes y sugerencias que ellas realizan y no optar por una posición autoritaria (de tipo patriarcal) al tratar de solucionar las distintas situaciones escolares, de manera unilateral y desde una única postura. 


\section{Referencias}

Alfaro Rojas, Susy, Montoya Tejos, Erick, Rodríguez Masís, Luis Carlos, Solano Gamboa, Alejandra y Solano Villalobos, Yesenia. (2003). Elaboración de un manual para la resolución constructiva de la etapa de iniciación laboral de docentes principiantes de educación primaria (tesis de licenciatura). Escuela de Orientación y Educación Especial, Facultad de Educación, Universidad de Costa Rica, San José, Costa Rica.

Arón Svigilsky, Ana María y Milicic, Neva. (2004). Clima social escolar y desarrollo personal. Un programa de mejoramiento. Santiago: Editorial Andrés Bello.

Bonilla Gamboa, Flory Stella. (1992). Orientación para cumplir las tareas adultas. Revista Educación, 16(1), 25-32.

Bustamante Carreño, María Teresa. (1997). La mujer y su constante crisis en busca del reconocimiento de su propia identidad. En Linda Berrón y Monserrat Sagot (comps.). Las mujeres y el poder. San José, Costa Rica: Editorial Mujeres.

Campos Vargas, Andrés, Castro Barrantes, María, Chavarría Molina, Yajaira, Gómez Gamboa, Luis Antonio, Ledezma Fonseca, Vivian y Quesada Rojas, Keylin. (2002). Promoción del desarrollo personal de los y las adolescentes escolarizados con discapacidad en el cantón de Montes de Oca (tesis de licenciatura). Escuela de Enfermería, Facultad de Educación, Universidad de Costa Rica, San José, Costa Rica.

Cornejo Abarca, José. (1998). Profesores que se inician en la docencia. Algunas reflexiones al respecto desde América Latina. Chile: Universidad Católica de Chile.

Delgadillo Solano, Ligia María. (1996). La mujer en la universidad: caso centroamericano. San José, Costa Rica: Guayacán.

Delgado, José Manuel y Gutiérrez Fernández, Juan. (1998). Métodos y técnicas cualitativas de la investigación en ciencias sociales. Madrid, España: Síntesis.

Gordillo Álvarez Valdés, María Victoria. (1988). Manual de Orientación Educativa. Madrid: Alianza.

Hershenson, David B. y Power, Paul W. (1987). Mental Health Counseling. USA: Allyn and Bacon.

Hidalgo Carmona, Carmen Gloria y Abarca Melo, Nureya. (2000). Comunicación interpersonal. Programa de entrenamiento en habilidades sociales. México: Ediciones ALFAOMEGA.

Jaramillo Loya, Horacio. (1997). El despertar del mago. México: Editorial Diana.

León de Leal, Magdalena. (1997). Poder y empoderamiento de las mujeres. Colombia: Fondo de Documentación Mujer y Género.

López Mojarro, Miguel. (2002). A la calidad por la evaluación. Barcelona: CISSPRAXIS. 
Orbach, Susie y Eichenbaum, Luise. (1989). Agridulce. México: Grijalbo.

Rodríguez Varela, Sandra. (2009). La autodeterminación de los proyectos de vida en mujeres en edad adulta media. (Tesis de Maestría). Posgrado en Gerontología. Universidad de Costa Rica, San José, Costa Rica.

Rogers Ramson, Carls. (1989). El camino del ser. Argentina: Kairós.

Smelzer J., Neil y Erikson H., Erik. (1983). Trabajo amor y poder en la vida adulta. Barcelona: Ediciones Grijalbo.

Tamayo Tamayo, Mario. (2002). El proceso de investigación científica. México: LIMUSA NORIEGA.

Taylor, S.J y Bogdan, Roberto. (1996). Introducción a los métodos cualitativos de investigación. España: Ediciones Paidós Ibérica.

Tonon del Toscano, Graciela. (2003). Calidad de vida y desgaste profesional: una mirada del síndrome del burnout. Buenos Aires, Argentina: Editorial Espacio.

Valles Martínez, Miguel. (1997). Técnicas cualitativas de investigación social. Reflexión metodológica y práctica profesional. Madrid: Síntesis. 